\title{
Influence of thermal treatment of nanometer-sized titanate and barium orthotitanate precursors on the electrorheological effect
}

\author{
K. V. Ivanov ${ }^{1}$, A. V. Agafonov ${ }^{1}$, A. E. Baranchikov ${ }^{2}$, V.K. Ivanov ${ }^{2}$, S. A. Kozyukhin ${ }^{2}$, \\ E. V. Fatyushina ${ }^{2}$, V. V. Kozik ${ }^{3}$ \\ ${ }^{1}$ G. A. Krestov Institute of Solution Chemistry, RAS, Ivanovo, Russia \\ ${ }^{2}$ Kurnakov Institute of General and Inorganic Chemistry, RAS, Moscow, Russia \\ ${ }^{3}$ National Research Tomsk State University, Tomsk, Russia \\ ava@isc-ras.ru
}

PACS 61.46.+w, 77.22.-d, 82.45.Vp, 82.80.-d

DOI 10.17586/2220-8054-2018-9-6-746-753

\begin{abstract}
The results for the sol-gel synthesis of acylate gels are reported in the titanium tetra-n-butoxide - barium hydroxide - acetic acid system at a molar ratio of $\mathrm{Ba}$ Ti components of $1: 1$ and $2: 1$. The thermal decomposition of these gels over temperatures ranging from $85-1200{ }^{\circ} \mathrm{C}$ was investigated using thermal analysis methods, XRD, FTIR spectroscopy, electron microscopy, and dielectrometry at frequencies from 25 $106 \mathrm{~Hz}$. The electrorheological effect in suspensions of powders synthesized at different temperatures with a stoichiometric Ba:Ti ratio $=1: 1$ and $2: 1$, in polydimethylsiloxane was studied. The influence of thermal gel treatment was analyzed on their dielectric properties as fillers of electrorheological fluids and on the magnitude of the electrorheological effect.
\end{abstract}

Keywords: sol-gel synthesis, barium titanate, electrorheological fluids, dielectric liquids, nanometer-sized, thermal treatment.

Received: 29 June 2018

Revised: 11 October 2018

\section{Introduction}

Electrorheological (ER) fluids are suspensions of polarizable semiconductor particles in dielectric liquids. The viscoplastic properties of ER fluids depend on the strength of the applied electric field up to the total loss of fluidity [1,2]. The loss of fluidity of the ER fluid in an electric field occurs due to polarization interactions, leading to structuring of the dispersed phase. The formation of chain or pillar structures from separate particles and closing of the interelectrode gap occurs within milliseconds. The presence of a limiting value of the electric field strength is typical for the electrorheological effect, after which the structure formation takes place and the fluid enhances its viscoplastic properties.

The magnitude of the ER effect is significantly affected by such factors as polarization, dielectric constant, conductivity, the applied electric field strength, the size, shape and structure of particles [3-9]. Another important factor affecting the properties of ER fluids is the water content in the dispersed phase [10]. Obviously, the aggregate functional properties of an ER fluid are determined by the composition and nature of the components.

The barium titanyl acylates complexes are considered promising among other fillers of ER fluids [11-14]. The yield strength of an ER fluid based on barium titanyl oxalate, with the surface of nanoparticles covered by adsorption layer of urea molecules, can exceed $100 \mathrm{kPa}$ in a $4 \mathrm{kV} / \mathrm{mm}$ field [15-17]. At the same time, the yield strength of barium titanate suspension obtained by calcination of acylate complexes in an electric field of $4 \mathrm{kV} / \mathrm{mm}$ does not exceed $1 \mathrm{kPa}$ [18]. Thus, the ER efficiency of the acylate complexes is significantly reduced during calcination. Apparently, this occurs as a result of polar organic group elimination from the material, leading to a decrease in the polarizability of the filler particles.

This work aims to reveal the influence of thermal treatment of acylate gels - the precursors of barium titanate and orthotitanate, used as ER fluid fillers, on the magnitude of the ER effect.

\section{Experimental section}

Barium hydroxide $\mathrm{Ba}(\mathrm{OH})_{2} \cdot 8 \mathrm{H}_{2} \mathrm{O}$, glacial acetic acid $\mathrm{CH}_{3} \mathrm{COOH}$ and $99 \%$ pure titanium tetra-n-butoxide $\mathrm{Ti}\left[\mathrm{O}\left(\mathrm{CH}_{2}\right)_{3} \mathrm{CH}_{3}\right]_{4}$ were used as starting materials. All reagents were purchased from SIGMA-Aldrich.

The following sol-gel synthesis of nanometer-sized barium titanyl acylate powders at stoichiometric Ba:Ti ratios of $1: 1$ and 2:1 was applied: $10 \mathrm{~g}$ of barium hydroxide octahydrate was dissolved in $17.31 \mathrm{~g}$ of glacial acetic acid at constant stirring until a clear solution was obtained, followed by dropwise addition of $10.8 \mathrm{~g}$ (or $5.4 \mathrm{~g}$ ) of titanium tetra-n-butoxide via a syringe. The contents of the reaction flask were stirred for an hour, then the temperature of the solution was raised to $85{ }^{\circ} \mathrm{C}$. After a short period of time, the solution changed into a 
transparent, durable gel. Drying the gel in air at $85{ }^{\circ} \mathrm{C}$ to a constant weight afforded white powders. According to elemental analysis, the content of organic phase in samples of dry gel powders with stoichiometric Ba:Ti ratios $=1: 1$ and 2:1 amounted to some 35 and $17 \%$ (with an accuracy of $1 \%$ ). The resulting powders were calcined for an hour in air at temperatures of $200,400,600,800$ and $1200{ }^{\circ} \mathrm{C}$ and used for subsequent analysis and for the preparation of ER fluids.

\section{Thermal evolution characterization of the obtained materials}

Transmission electron microscopy (electron microscope $2 \mathrm{MB}-100 \mathrm{~L}$ ) was used to study the shape and particle size of the powders obtained. According to electron microscopy, when the Ba:Ti molar ratio in the synthesis is $1: 1$, the $50-200 \mathrm{~nm}$ polydisperse particles are formed, while an initial 2:1 Ba:Ti ratio affords $50-80 \mathrm{~nm}$ polydisperse particles (Fig. 1). From the electron microscopic images it can be seen that the particles have a rounded shape.

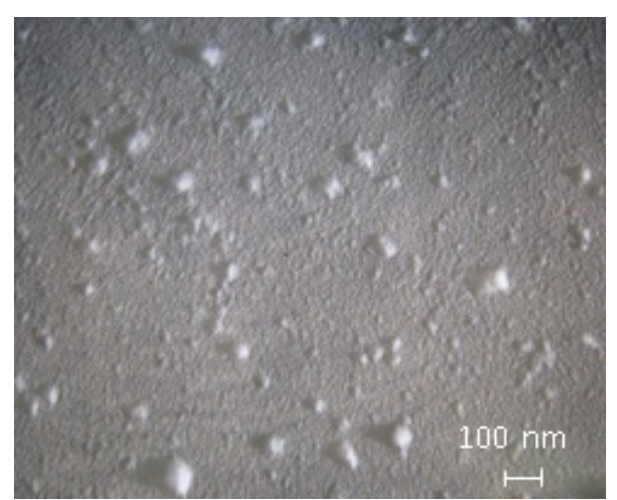

(a)

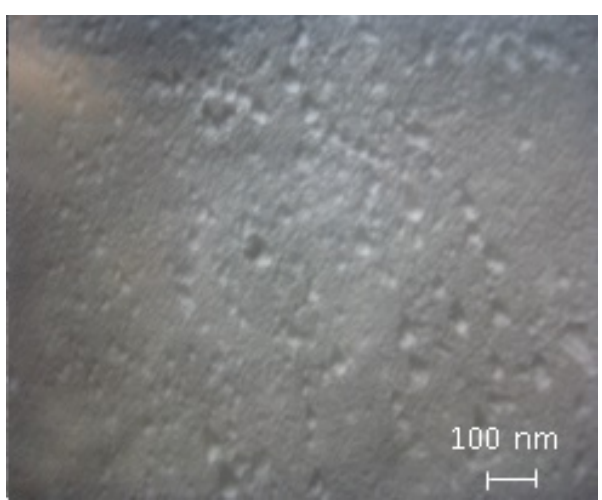

(b)

FIG. 1. TEM images of dried at $85{ }^{\circ} \mathrm{C}$ acylate gels obtained at Ba:Ti 1:1 (a) and 2:1 (b) molar ratios

The analysis of phase composition was performed on a DRON-2 diffractometer using $\mathrm{Cu}-\mathrm{K} \alpha$ radiation. According to the results of X-ray diffraction, when the initial stoichiometric Ba:Ti ratio is 1:1 (Fig. 2(a)), the formation of barium titanate starts at $600{ }^{\circ} \mathrm{C}$ and terminates at $800{ }^{\circ} \mathrm{C}$. When the initial Ba:Ti molar ratio of components is 2:1 (Fig. 2(b)), the reflexes corresponding to barium titanate and barium orthotitanate are observed at $400{ }^{\circ} \mathrm{C}$. With an increase of temperature to $1200{ }^{\circ} \mathrm{C}$, the reflexes of $\mathrm{BaTiO}_{3}$ and $\mathrm{Ba}_{2} \mathrm{TiO}_{4}$ are observed.

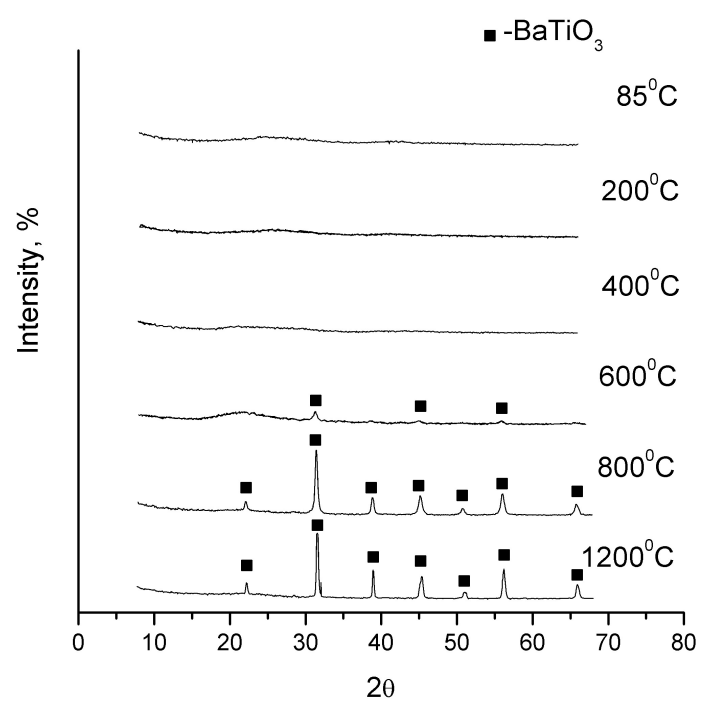

(a)

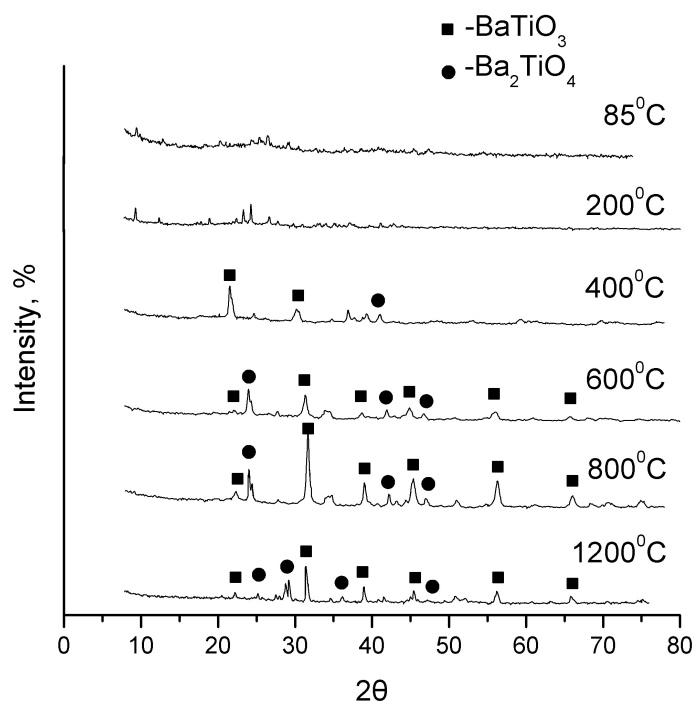

(b)

FIG. 2. X-ray phase analysis of barium-titanium acylate complexes obtained at Ba:Ti 1:1 (a) and $2: 1$ (b) molar ratios and their calcination products at various temperatures 
The IR spectra of the synthesized compounds were recorded in a potassium bromide matrix on a VERTEX 80v FT-IR spectrometer. IR spectra of synthesized ultradispersed materials (Fig. 3) contain absorption bands characteristic for barium acetate, titanium dioxide and its hydroxy-forms, butoxy groups, as well as titanium butoxide and water molecules: $\mathrm{Ba}-\mathrm{OH} 1459,1420$ and $1379 \mathrm{~cm}^{-1}$; titanium butoxide $1458,1429,1371$ and $1362 \mathrm{~cm}^{-1}$. The band at $1125 \mathrm{~cm}^{-1}$ can be attributed to the vibrations of the Ti-O-C bond, the band at $948 \mathrm{~cm}^{-1}-$ to Ti-OH vibrations, and the band at $3390 \mathrm{~cm}^{-1}$ may correspond to the stretching of $\mathrm{OH}$ groups. The $\mathrm{CO}_{3}^{2-}$ ion has a characteristic absorption maximum at $1540 \mathrm{~cm}^{-1}$, and the band at $1461 \mathrm{~cm}^{-1}$ characterizes asymmetric oscillations in the stretching of the $\mathrm{C}-\mathrm{O}$ bond. For the acetate ion, depending on the type of coordination, absorption maxima for symmetric $(\nu S)$ and antisymmetric $(\nu A) \mathrm{C}-\mathrm{O}$ vibrations are observed at different positions [19] for monodentate $\left(\nu S=1295 \mathrm{~cm}^{-1}, \nu A=1720 \mathrm{~cm}^{-1}\right)$, bridged bidentate $\left(\nu S=1430 \mathrm{~cm}^{-1}, \nu A=1590 \mathrm{~cm}^{-1}\right)$ and chelate bidentate complexes $\left(\nu S=1470 \mathrm{~cm}^{-1}, \nu A=1550 \mathrm{~cm}^{-1}\right)$. An intense absorption band at $650 \mathrm{~cm}^{-1}$ characterizes the vibrations of the Ti-O-Ti bond. The Ba-O oscillations correspond to the band at $474 \mathrm{~cm}^{-1}$. As the samples are thermally processed, the IR spectra begin to correspond to the spectra of the corresponding titanates.

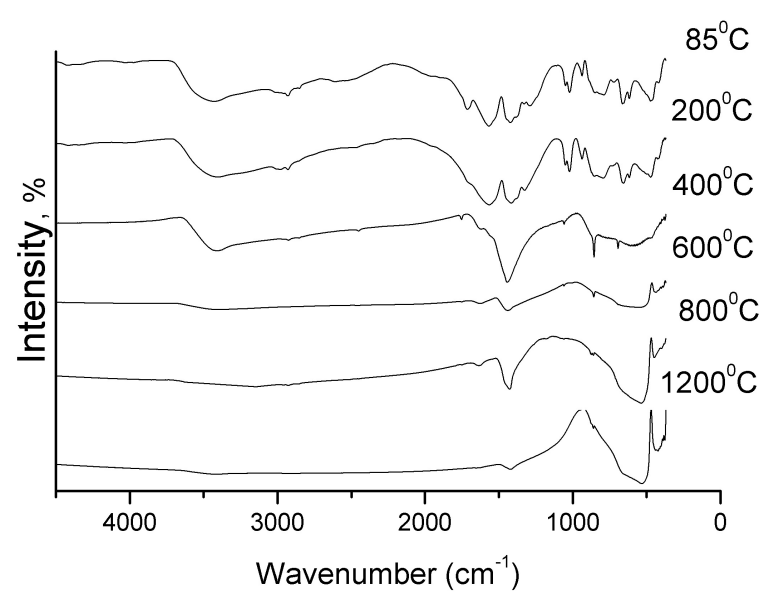

(a)

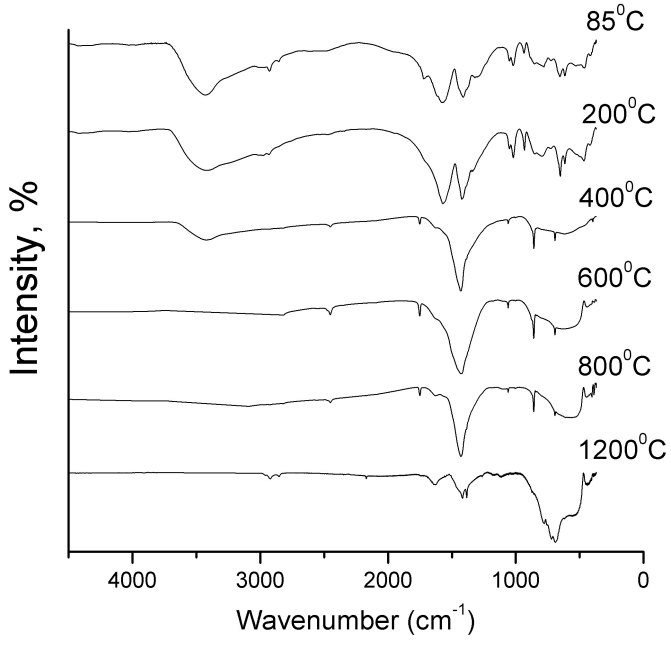

(b)

FIG. 3. IR spectra of acylate complexes powders with Ba:Ti 1:1 (a) and 2:1 (b) molar ratios and their calcination products at different temperature

The thermal analysis of the synthesized powders was performed on a 1000D Paulik-Paulik-Erdei derivatograph at a heating rate of $5{ }^{\circ} \mathrm{C} / \mathrm{min}$. One can distinguish three ranges associated with a change in the mass of the sample on the thermograms of synthesized materials (Fig. 4). The first range $\left(50-150{ }^{\circ} \mathrm{C}\right)$ might be associated with the removal of acetic acid and the adsorbed water. The second range $\left(150-450{ }^{\circ} \mathrm{C}\right)$ corresponds to the decomposition of titanium butoxide complexes with acetate ions to titanium dioxide. At temperatures of $500-750{ }^{\circ} \mathrm{C}$ the thermal decomposition of barium carbonate, which is present as an impurity in the material, is likely to occur with the formation of barium titanate. The total weight loss for each of the samples does not exceed $45 \%$.

The analysis of materials calcined at $1200{ }^{\circ} \mathrm{C}$ with differential scanning calorimetry (DSC) was performed on a DSC 204 F1 Phoenix calorimeter with the $\mu$-sensor. The maxima on the heat capacity-temperature curves, which indicate a phase transition, are observed at temperatures ranging from $0-200{ }^{\circ} \mathrm{C}$ for both materials with $1: 1$ and 2:1 Ba:Ti molar ratio. For the system with a stoichiometric 1:1 Ba:Ti molar ratio, the phase transition temperature is $119{ }^{\circ} \mathrm{C}$ and for the system with a $2: 1 \mathrm{Ba}$ :Ti ratio, the phase transition temperature is $122{ }^{\circ} \mathrm{C}$ (Fig. 5).

The dielectric spectra of $30 \%$ suspensions of the resulting powders in silicone oil PMS-20 were measured in a cylindrical cell of the condenser type at $T=293 \mathrm{~K}$ using an E7-20 immittance meter in the frequency range from 25 to $1 \times 106 \mathrm{~Hz}$. Fig. 6 shows the dependences of $\varepsilon$ and $\tan \delta$ on the frequency of the electric current.

The $\tan \delta$ frequency dependences of suspensions indicate the relaxation character of the dielectric spectra. As it follows from Fig. 6(a), a suspension of the initial powder is characterized by the presence of a relaxation maximum, which disappears after calcination. As is evident from the Fig. 6(b), the position of relaxation maximum for the suspension of the initial powder with the 2:1 Ba:Ti ratio is at lower frequency in comparison to the powder with the 1:1 Ba:Ti ratio. In that regard, the relaxation shape of the dielectric spectrum persists for suspensions with filler particles calcined at 200 and $400{ }^{\circ} \mathrm{C}$. An increase in the temperature of heat treatment of fillers with 


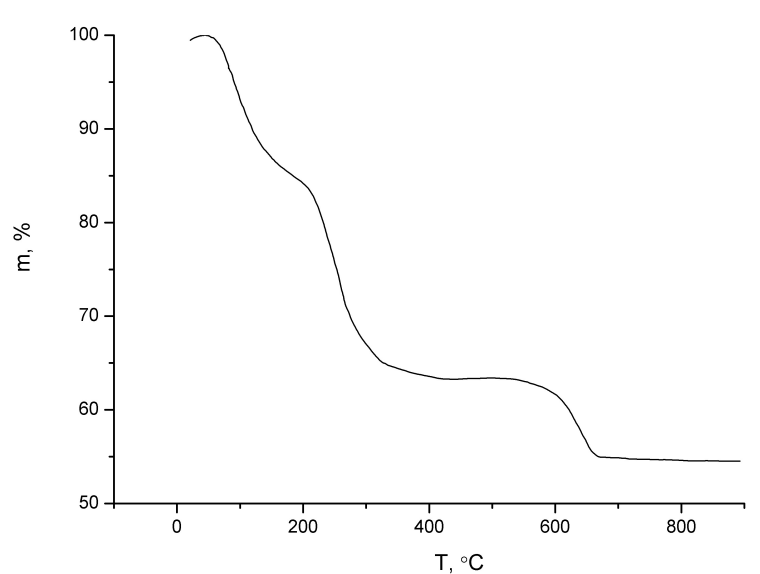

(a)

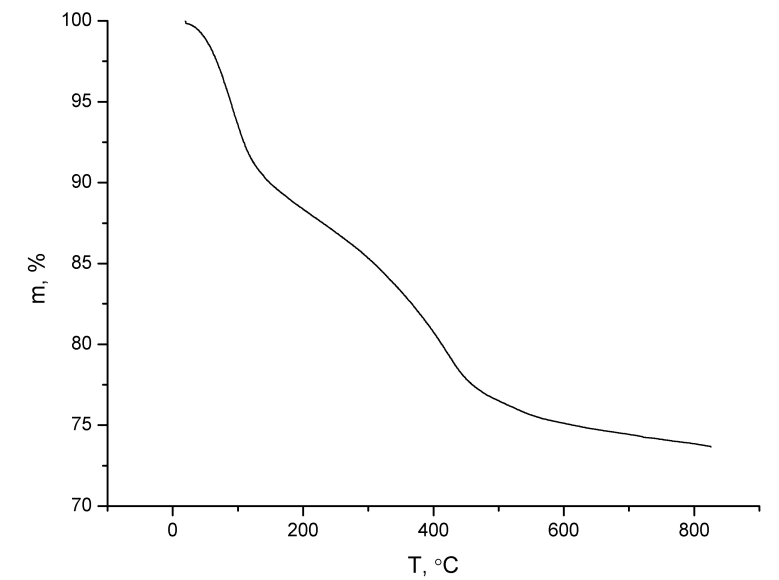

(b)

FIG. 4. Thermal analysis of acylate complexes powders with Ba:Ti 1:1 (a) and 2:1 (b) molar ratios

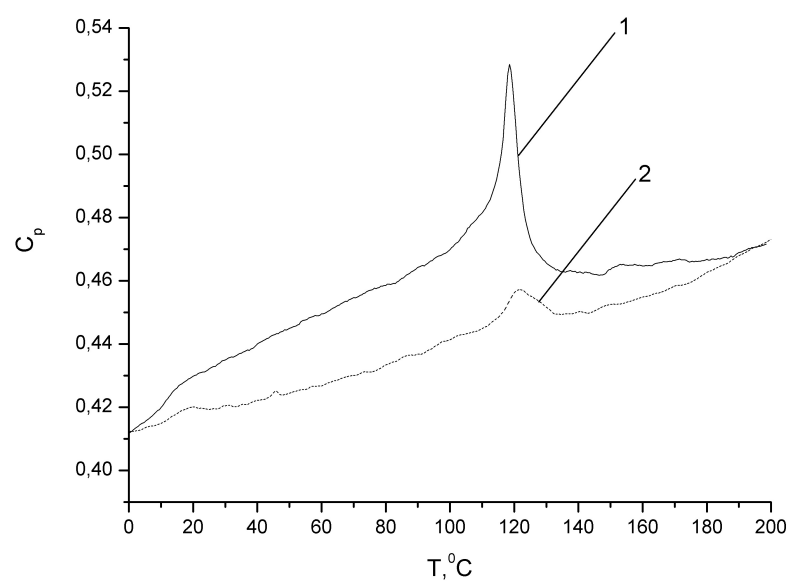

FIG. 5. DSC of materials obtained by calcination at $1200{ }^{\circ} \mathrm{C}$ with Ba:Ti 1:1 (1) and 2:1 (2) molar ratios

$\mathrm{BaO}: \mathrm{TiO}_{2}$ ratios of $1: 1$ and $2: 1$ results in a similar shape of $\varepsilon$ and $\tan \delta$ frequency dependences for these systems and an absence of relaxation maxima in them.

\section{Results and discussion}

The obtained complex of data characterizing the phase composition of the obtained materials at different temperatures allows us to make assumptions about the patterns of structure formation during its thermal treatment.

The interaction of titanium tetra-n-butoxide with glacial acetic acid results in the gelation due to the formation of polynuclear complexes in which bidentate acetate ions act as ligands (Fig.7) [20]. When barium hydroxide is dissolved in glacial acetic acid, barium acetate forms, which, apparently, can be incorporated into the structure of the titanium acylate complex [21] or form clathrate complexes [22].

The thermal treatment of the obtained powders with a different ratio of reagents in the mixture leads to their decomposition. Thermal decomposition of the acylate polynuclear complex of titanium tetra-n-butoxide in an oxidizing atmosphere is accompanied by the removal of volatile organic components, oxidation of acetate and butoxide groups. In parallel, the decomposition of barium acetate occurs, which is accompanied by successive transformations [21]:

$$
\mathrm{Ba}\left(\mathrm{OOCCH}_{3}\right)_{2} \rightarrow \mathrm{BaCO}_{3}+\mathrm{CO} \rightarrow \mathrm{BaO}+\mathrm{CO}_{2} \text {. }
$$

In general, thermal transformations of materials obtained from precursors with the 1:1 and 2:1 Ba:Ti ratios involve the same processes, however, product formation proceeds according to different schemes. To a considerable degree, this is determined by both the reactivity of barium orthotitanate and by the various molar ratios of water and titanium butoxide in the synthesis of $\mathrm{Ba}_{2} \mathrm{TiO}_{4}$ and $\mathrm{BaTiO}_{3}$. As follows from Figs. 2(a), 3(a) and 4(1), in a 


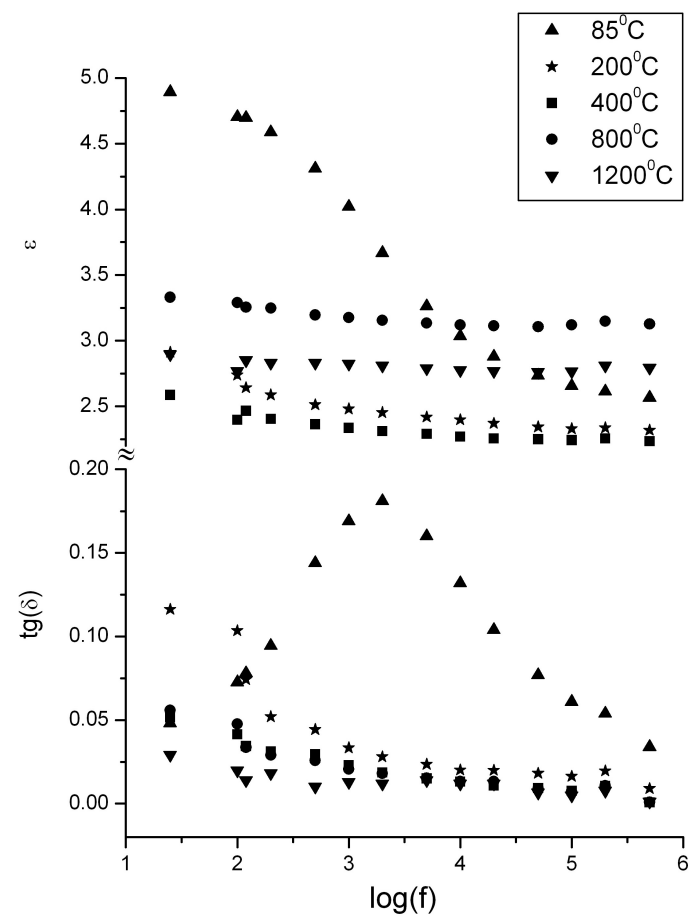

(a)

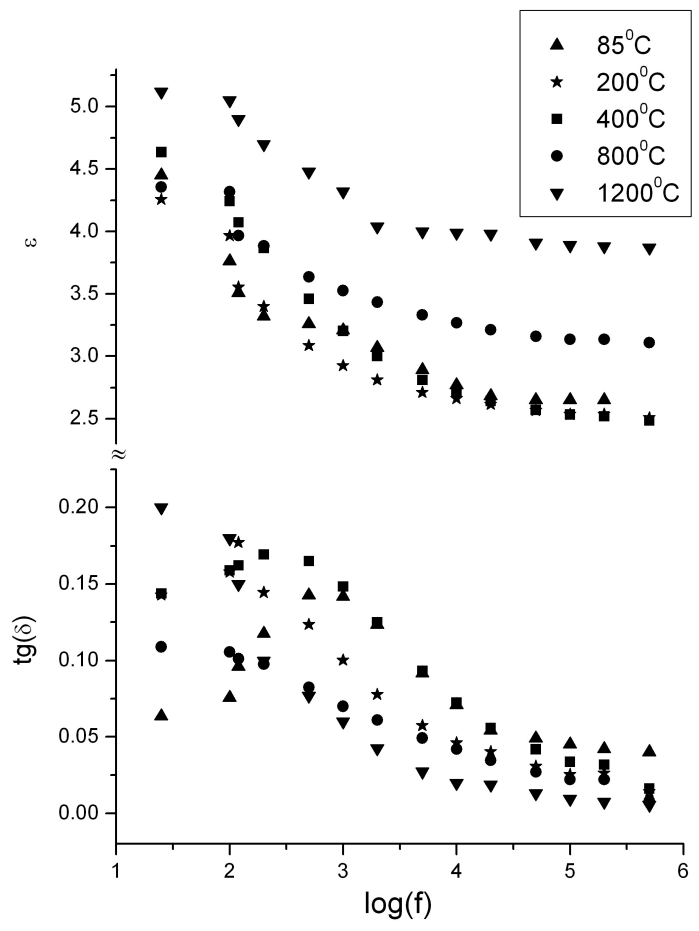

(b)

FIG. 6. Dielectric parameters of $30 \%$ suspensions of samples with Ba:Ti 1:1 (a) and 2:1 (b) stoichiometric ratio in silicone oil at $25{ }^{\circ} \mathrm{C}$

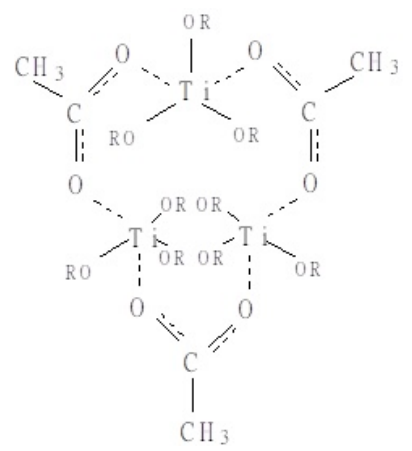

FIG. 7. Polynuclear acylate complex of titanium tetrabutoxide ( [20])

system containing $\mathrm{Ba}$ and $\mathrm{Ti}$ in a ratio of $1: 1$ at 200 and $400{ }^{\circ} \mathrm{C}$, the formation of $\mathrm{X}$-ray amorphous products containing organic residues occurs as well as hydroxy compounds of these metals. At that the formation of $\mathrm{BaTiO}_{3}$ phase begins at $600{ }^{\circ} \mathrm{C}$. This process is accompanied by the decomposition of barium carbonate: $\mathrm{BaCO}_{3} \rightarrow \mathrm{BaO}+$ $\mathrm{CO}_{2}$ and the reaction $\mathrm{BaO}+\mathrm{TiO}_{2} \rightarrow \mathrm{BaTiO}_{3}$. At $1200{ }^{\circ} \mathrm{C}, \mathrm{BaTiO}_{3}$ is present in the system as well as traces of $\mathrm{BaO}$.

The thermal evolution of the product obtained from starting material with 2:1 Ba:Ti ratio proceeds according to a different scheme. As follows from Figs. 2(b), 3(b) and 4(2), calcination at $200{ }^{\circ} \mathrm{C}$ does not lead to significant changes in the physicochemical characteristics of the material.

The phases of $\mathrm{BaTiO}_{3}$ and $\mathrm{Ba}_{2} \mathrm{TiO}_{4}$ start to form at $400{ }^{\circ} \mathrm{C}$. This is $200{ }^{\circ} \mathrm{C}$ below the temperature of the $\mathrm{BaTiO}_{3}$ phase formation in the synthesis with the 1:1 Ba:Ti ratio. We explain such differences by different ratios of $\mathrm{Ba}: \mathrm{Ti}$ and $\mathrm{H}_{2} \mathrm{O}: \mathrm{Ti}$ in the reaction mixtures. Calcination at 600,800 and $1200{ }^{\circ} \mathrm{C}$ favors an increase in the phase content of $\mathrm{BaTiO}_{3}$ and $\mathrm{Ba}_{2} \mathrm{TiO}_{4}$. The product calcined at $1200{ }^{\circ} \mathrm{C}$ is a mixture of $\mathrm{Ba}_{2} \mathrm{TiO}_{4}$ and $\mathrm{BaTiO}$ 作 a ratio of $30 \%$ and $70 \%$.

The $\mathrm{Ba}_{2} \mathrm{TiO}_{4}$ and $\mathrm{BaTiO}_{3}$ phases have different $\mathrm{Ba}$ :Ti stoichiometric ratios. This affects the packing density of ions in the $\mathrm{Ba}_{2} \mathrm{TiO}_{4}$ and $\mathrm{BaTiO}_{3}$ lattices and determines the polarization parameters of the materials. Barium 
titanate with a tetragonal structure at room temperature $\left(\sim 25^{\circ} \mathrm{C}\right)$ has a dielectric constant of about 1500 , and barium orthotitanate with an orthorhombic structure of about $20^{\circ} \mathrm{C}$ has a dielectric constant of about 1500 [19].

The described transformations of the acylate complexes obtained at different Ba:Ti ratios are reflected in the corresponding dielectric spectra of the powder suspensions in silicone oil PMS-20.

The influecne of thermal treatment of powders on the ER effect was determined from the change in shear stress, as a function of the applied DC electric field in the range $0-7 \mathrm{kV} / \mathrm{mm}$. For the investigation, $30 \%$ suspensions of the obtained materials in polydimethylsiloxane (PMS-20 Penta Silicones) were prepared. The required amount of powder was triturated in polydimethylsiloxane until a homogeneous suspension was formed. The measurements were carried out on a modified Brookfield DV-II viscosimeter at various shear rates and DC electric field strengths applied to a $1 \mathrm{~mm}$ parallel plane gap between the electrodes.

The ER effect theoretical models, based on the electrostatic theory of dipole-dipole interactions, describe the attractive force between the particles in an electric field according to the following equation [23]:

$$
F=\frac{24 a^{6} \varepsilon_{0} E^{2}}{R^{4}} \frac{K_{f}\left(K_{p}-K_{f}\right)^{2}}{\left(K_{p}+2 K_{f}\right)^{2}},
$$

where $a$ - is radius of the filler particle, $R$ - interparticle distance, $\varepsilon_{0}$ - dielectric constant of vacuum, $E-$ the electric field strength, $K_{f}$ - dielectric permittivity of a liquid dielectric (dispersion medium), $K_{p}-$ dielectric constant of the filler particle material.

This equation emphasizes the role of the differences in the dielectric parameters of the dispersion medium and the dispersed phase in the formation of the ER effect and justifies the quadratic dependence of the electrorheological effect on the electric field strength.

The dependence of the shear stress on the electric field strength at various shear rates for suspensions of acylate complexes with the $1: 1$ and 2:1 Ba:Ti ratio of components dried at $85{ }^{\circ} \mathrm{C}$ are presented in Fig. 8. The results demonstrated in Fig. 8(b) obey the equation 1, while the results in Fig. 8(a) do not obey. A direct proportion of the shear stress on the field strength is characteristic for acylate complexes with a Ba:Ti ratio of 1:1 after saturation is achieved after $E=4.5 \mathrm{kV} / \mathrm{mm}$ starting from a field strength of $0.5 \mathrm{kV} / \mathrm{mm}$. This may be due to presence of the adsorbed acetic acid on the surface of particles, which leads to the effects reported in [24].

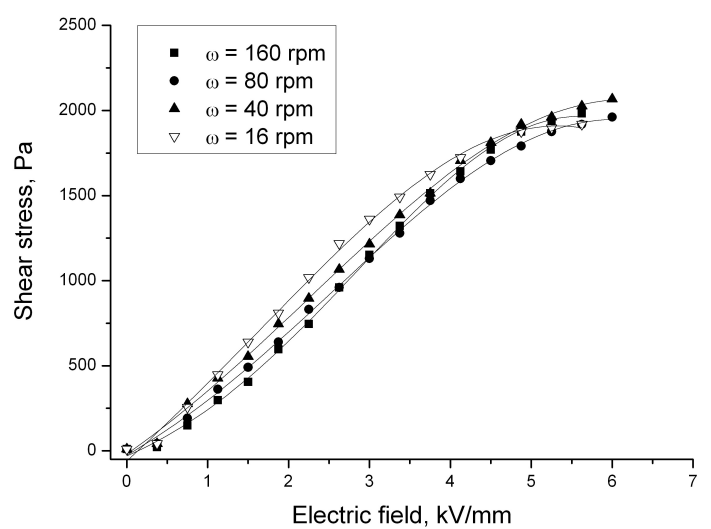

(a)

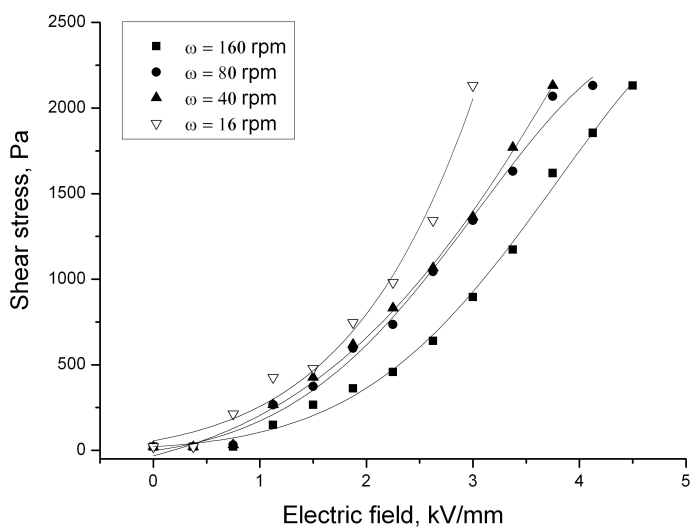

(b)

FIG. 8. The dependence of shear stress on the electric field strength at different shear rates for $30 \%$ suspensions of acylate complexes powders dried at $85^{\circ} \mathrm{C}$. 1:1 (a) and 2:1 (b) Ba:Ti ratios

The influence of the filler calcination temperature on the magnitude of the ER effect at a shear rate of $160 \mathrm{rpm}$ is shown in Fig. 9. One can note that the ER effect regularities associated with the use of filler materials subjected to different thermal treatments can be explained from the standpoint of the classic electrostatic theory of the dipoledipole interactions. As follows from Fig. 6, the permittivity of the suspensions is successively decreased with the increasing calcination temperature. In accordance with equation 1, this leads to a decrease in the attractive force between the filler particles and to a decrease in the ER effect, which is observed (Fig. 9).

In the process of ER fluids structuring in electric fields with increasing field strength the distance between particles decreases (a decrease in the thickness of the dielectric liquid interlayer between the particles, the appearance of tight contacts), leading to an increase in conductivity. In this case, the conductivity increases proportionally to the number of formed contacts between the filler particles. The voltammetric parameters for $30 \%$ suspensions of materials with 1:1 and 2:1 Ba:Ti ratios calcined at different temperatures at a shear rate of $160 \mathrm{rpm}$ are significantly 


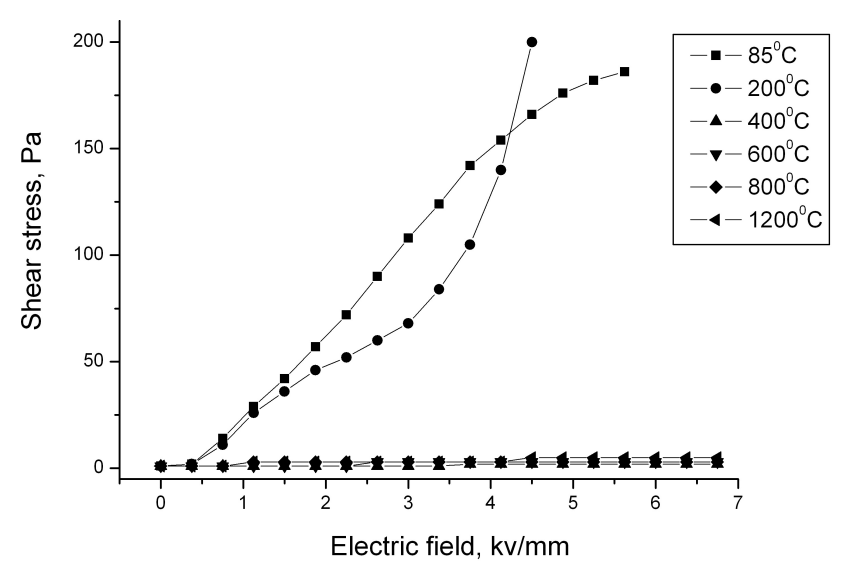

(a)

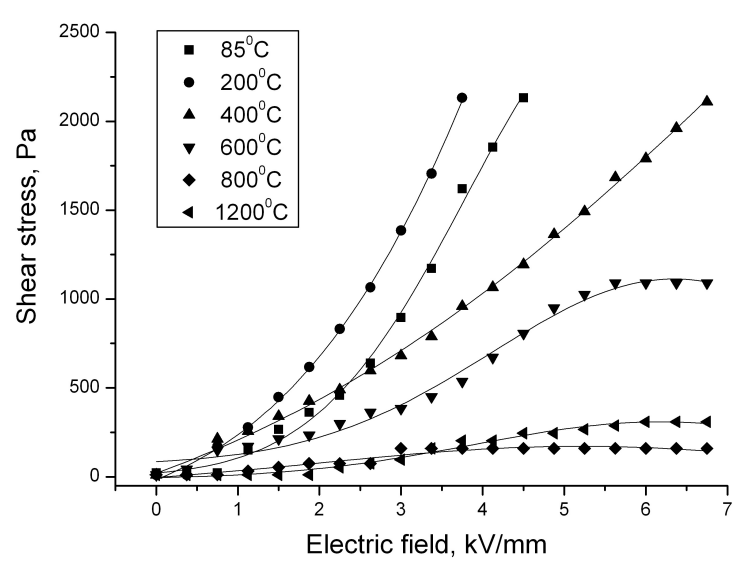

(b)

FIG. 9. Dependence of the shear stress of $30 \%$ suspensions of acylate complexes with the 1:1 (a) and 2:1 (b) Ba:Ti ratios calcined at different temperatures on the electric field strength at a shear rate of $160 \mathrm{rpm}$

different (Fig. 10). For $30 \%$ suspensions of materials with 1:1 Ba:Ti ratio, an increase in the calcination temperature leads to a consistent decrease in the current flowing through the suspension at an equal applied voltage. This may be due to the fact that molecules of acetic acid and polar acetate groups are present on the surface of filler particles that determine the value of the conductivity. As the organic components are removed, the conductivity of the suspensions decreases. For $30 \%$ suspensions of materials with 2:1 Ba:Ti ratio (Fig. 1(b)), an increase in the calcination temperature of the filler material leads to an increase in the electrical conductivity of the suspensions. From Fig. 6, one can see that the value of the dielectric loss tangent for such suspensions is higher than for the systems with the $1: 1 \mathrm{Ba}$ Ti ratio.

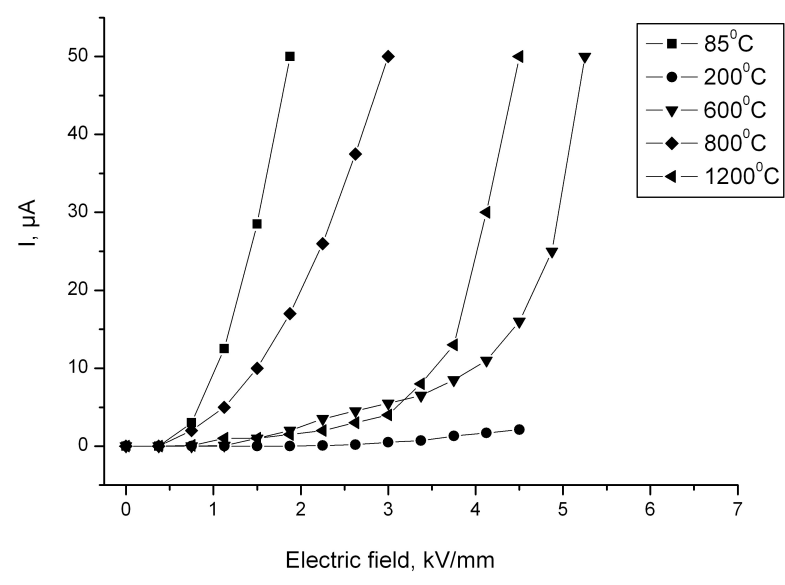

(a)

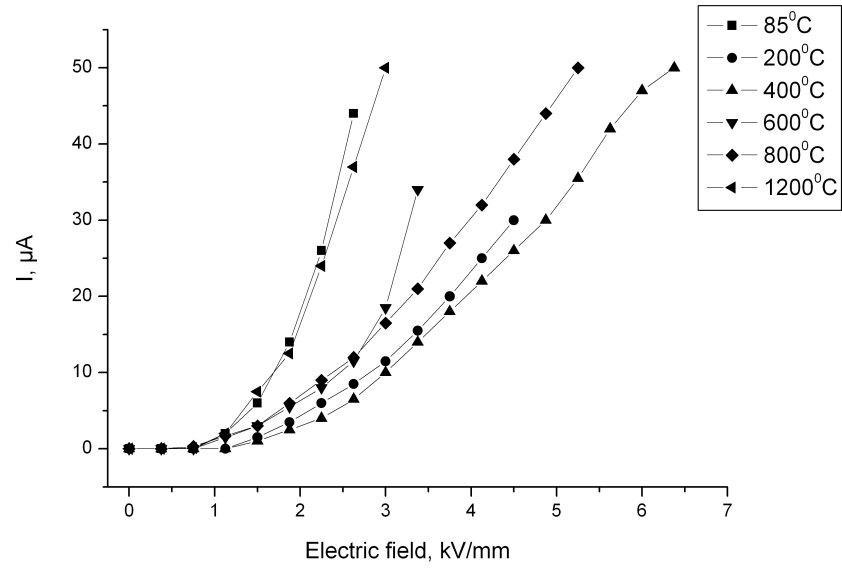

(b)

FIG. 10. Voltammetric parameters for $30 \%$ suspensions of acylate complexes with the 1:1 (a) and 2:1 (b) Ba:Ti ratios calcined at different temperatures at a shear rate of $160 \mathrm{rpm}$

\section{Conclusions}

The acylate gels were obtained via a sol-gel method in the titanium tetra-n-butoxide-barium hydroxide-acetic acid system in excess of acetic acid with a molar ratio of Ba:Ti components of 1:1 and 2:1. The regularities for the formation of $\mathrm{Ba}_{2} \mathrm{TiO}_{4}$ and $\mathrm{BaTiO}_{3}$ phases during calcination of the obtained gels at 200, 400, 600, 800 and $1200{ }^{\circ} \mathrm{C}$ were investigated. A detailed study of phase transformations for the obtained materials was performed using thermogravimetric analysis, differential scanning calorimetry, X-ray phase analysis, FTIR spectroscopy, and electron microscopy. It was established that the thermolysis of acylate complexes with 1:1 Ba:Ti ratio at 200 and $400{ }^{\circ} \mathrm{C}$ affords X-ray amorphous products with a low organic phase content and significant metal hydroxy 
compounds content. The formation of the $\mathrm{BaTiO}_{3}$ phase begins at $600{ }^{\circ} \mathrm{C}$, and at $1200{ }^{\circ} \mathrm{C}, \mathrm{BaTiO}_{3}$ is present in the system with traces of $\mathrm{BaO}$. The decomposition of the acylate complexes with the 2:1 $\mathrm{Ba}$ :Ti ratio leads to the formation of $\mathrm{BaTiO}_{3}$ and $\mathrm{Ba}_{2} \mathrm{TiO}_{4}$ phases as low as at $400{ }^{\circ} \mathrm{C}$. This is $200{ }^{\circ} \mathrm{C}$ lower than the temperature of $\mathrm{BaTiO}_{3}$ phase formation in the synthesis with $1: 1 \mathrm{Ba}$ :Ti ratio. Calcination at 600,800 and $1200{ }^{\circ} \mathrm{C}$ leads to a gradual increase of the $\mathrm{Ba}_{2} \mathrm{TiO}_{4}$ and $\mathrm{BaTiO}_{3}$ phase content in the product. The product calcined at $1200{ }^{\circ} \mathrm{C}$ is a mixture of the $\mathrm{Ba}_{2} \mathrm{TiO}_{4}$ and $\mathrm{BaTiO}_{3}$ phases in a ratio of $30 \%$ and $70 \%$, respectively. For the first time, data was obtained on the influence of thermal treatment temperature on the magnitude of the ER effect in suspensions based on these materials. It was established that the regularities of the ER effect magnitude variation can be explained from the standpoint of the classical electrostatic theory of the dipole-dipole interactions in electric fields.

\section{References}

[1] Agafonov A.V., Zakharov A.G. Electrorheological fluids. Russ. J. Gen. Chem., 2010, 80, P. 567-575.

[2] Liu Y.D., Choi H.J. Electrorheological fluids: smart soft matter and characteristics. Soft Matter, 2012, 8, P. 11961-11978.

[3] Wen W., Huang X., Sheng P. Electrorheological fluids: structures and mechanisms. Soft Matter, 2008, 4, P. $200-210$.

[4] Wu C.W., Conrad H. Dielectric and conduction effects in non-Ohmic electrorheological fluids Phys. Rev. E, 1997,56 (5), P. $5789-5797$.

[5] Whitte M., Bullough W.A., Peel D.J., et al. Dependence of electrorhrologycal response on conductivity and polarization time. Phys. Rev. E, 1994, 49 (6), P. 5249-5283.

[6] Agafonov V., Kraev A.S., et al. Comparative study of the electrorheological effect in suspensions of needle-like and isotropic cerium dioxide nanoparticles. Rheol Acta, 2018, 57, P. 307-315.

[7] Agafonov A.V., Kraev A.S., et al. Properties of electrorheological fluids based on nanocrystalline cerium dioxide. Russ. J. Inorg. Chem., 2017, 62, P. 625-632.

[8] Agafonov A.V., Krayev A.S., et al. Nanocrystalline ceria: a novel material for electrorheological fluids. $R S C A d v$., 2016, 6, P. 88851-88858.

[9] Parthasarathy M., Klingenberg D.G. Electrorheology: mechanisms and models. Mater. Sci. Eng., 1996, 17, P. 57-103.

[10] Ivanov K.V., Ivanova O.S., Agafonov A.V., Kozyukhin S.A. The dielectric properties and flow of electrorheological fluids based on polymer-coated nanodispersed barium tetraacetate titanyl particles upon a dynamic shear in electric fields. Colloid Journal, 2017, 79 (2), P. 204-211.

[11] Xufeng Dong, Ning Ma, et al. The contribution of friction to electrorheological properties of a chrysanthemum-like particle suspension. $R S C A d v$., 2015, 5, P. 74656-74663.

[12] Wu J., Song Z., et al. Giant electrorheological fluids with ultrahigh electrorheological efficiency based on a micro/nano hybrid calcium titanyl oxalate composite. NPG Asia Materials, 2016, 8, P. 322.

[13] Liu Y., Guan J., et al. Chromium doped barium titanyl oxalate nano-sandwich particles: A facile synthesis and structure enhanced electrorheological properties. Materials Chemistry and Physics, 2010, 122, P. 73-78.

[14] Jiang W., Jiang C., Gong X., Zhang Z. Structure and electrorheological properties of nanoporous $\mathrm{BaTiO}_{3}$ crystalline powders prepared by solgel method. J. Sol-Gel Sci. Technol., 2009, 52, P. 8-14.

[15] Wen W., Huang X., et al. The giant electrorheological effect in suspensions of nanoparticles. J. Nature Materials, 2003, 2, P. 727-730.

[16] Bose H., Trendler A. Comparison of rheological and electric properties of ER fluids based on different materials. Int. J. Mod. Phys. B, 2001, 15 (6-7), P. 626-633.

[17] Rankin P.J., Klingenberg D.J. The electrorheology of barium titanate suspensions. J. Rheol., 1998,42 (3), P. $639-656$.

[18] Ahmad T., Ganguli A.K. Synthesis of nanometer-sized particles of barium orthotitanate prepared through a modified reverse micellar route: Structural characterization, phase stability and dielectric properties. J. Mater. Res., 2004,19 (10), P. $2905-2912$.

[19] Schubert U. Organically modified transition metal alkoxides: Chemical problems and structural issues on the way to materials syntheses. Acc. Chem. Res., 2007, 40, P. 730-737.

[20] Wang L., Liu L., et al. Wet routes of high purity $\mathrm{BaTiO}_{3}$ nanopowders. Journal of Alloys and Compounds, $2007,440, \mathrm{P} .78-83$.

[21] Hwang U.Y., Park H.S., Koo K.K. Behavior of barium acetate and titanium isopropoxide during the formation of crystalline barium titanate. Ind. Eng. Chem. Res., 2004, 43, P. 728-734.

[22] Hao T. Electrorheological fluids: The non-aqueous suspensions. Cambridge Massachusetts USA, 2005, 22, 553 p.

[23] Shen R., Wang X., et al. Polar-molecule-dominated electrorheological fluids featuring high yield stresses. Adv. Mater., 2009, 21, P. 46314635.

[24] Agafonov A.V., Davydova O.I., et al. Unexpected effects of activator molecules' polarity on the electroreological activity of titanium dioxide nanopowders. J. Phys. Chem. B, 2017, 121 (27), P. 6732-6738. 